\title{
PATHOLOGICAL AND VIROLOGICAL ANALYSES OF SEVERE ACUTE RESPIRATORY SYNDROME- ASSOCIATED CORONAVIRUS INFECTIONS IN EXPERIMENTAL ANIMALS
}

\author{
Noriyo Nagata, Naoko Iwata, Hideki Hasegawa, Yasuko Asahi-Ozaki, \\ Yuko Sato, Ayako Harashima, Shigeru Morikawa, Masayuki Saijo, \\ Shigeyuki Itamura, Takehiko Saito, Takato Odagiri, Masato Tashiro, \\ Yasushi Ami, and Tetsutaro Sata*
}

\section{INTRODUCTION}

Severe acute respiratory syndrome (SARS) is a recently identified emerging infectious disease caused by SARS-associated coronavirus (SARS-CoV). To determine the pathological features of SARS-CoV infection in experimental animals, its clinical, pathological, and virological features were investigated in cynomolgus monkeys, BALB/c mice, and F344 rats. The susceptibility of these animals to SARS-CoV infection was evaluated to identify suitable animal models for studies of the pathogenesis and treatment of SARS.

\section{MATERIALS AND METHODS}

The SARS-CoV, HKU39849 isolate was used in the present study. ${ }^{1}$ The virus was propagated three times in Vero E6 cells, and the infectious doses of the virus stock were expressed as the $50 \%$ tissue culture infective dose $\left(\mathrm{TCID}_{50}\right)$ on these cells. Three-year-old male cynomolgus monkeys (Macaca fascicularis), 4-week-old female BALB/c mice, and 4-week-old F344 rats were used. Monkeys were inoculated intranasally with $10^{3}$ or $10^{6}$ TCID $_{50}$ of SARS-CoV in $3.5 \mathrm{ml}$ of medium, or intratracheally with $10^{8}$ TCID $_{50}$ in $5 \mathrm{ml}$ of medium. BALB/c mice and F344 rats were inoculated intranasally with $2 \times 10^{6} \mathrm{TCID}_{50}$ of SARS-CoV in $20 \mu \mathrm{l}$ of medium and $10^{7}$ TCID $_{50}$ in $100 \mu 1$ of medium, respectively. After inoculation, these animals were observed for clinical symptoms and sacrificed for pathological examination. Virus isolation and viral infectivity titers were investigated in

* National Institute of Infectious Diseases, Musashimurayama, Tokyo 208-0011, Japan. 
Vero E6 cell cultures. The SARS-CoV genome quantified by one-step reverse transcription and quantitative PCR assay using a LightCycler SARS-CoV quantification kit (Roche Diagnostics, Indianapolis, IN). All procedures in which infectious SARS-CoV was manipulated were carried out under biosafety level 3 conditions. The National Institute of Infectious Diseases Animal Care and Use Committee approved the animal studies to be carried out in an animal biosafety level 3 facility.

\section{RESULTS}

In monkeys, following intranasal inoculation with $10^{6} \mathrm{TCID}_{50}$ of SARS-CoV, the virus was isolated from throat and nasal swabs, and the viral genome was detected in rectal swabs collected between 2 and 7 days postinoculation (p.i.). In one of the two monkeys inoculated intratracheally with $10^{8}$ TCID $_{50}$ of SARS-CoV, virus and viral genome were detected in throat swabs collected on day 2 p.i. and in rectal swabs collected from days 4 to 7 p.i., respectively. Virus antigen-positive alveolar cells and macrophages were detected in the lower lobes of the lungs on day 7 p.i. in monkeys after intratracheal inoculation (Figure 1A). Angiotensin-converting enzyme 2 (ACE2, a receptor for SARS-CoV ${ }^{2}$ antigen-positive cells were observed in the virus-infected area and were repairing swelled type II alveolar epithelium in the lung of monkeys (Figure $1 \mathrm{~A}, \mathrm{~B}$, and C). In BALB/c mice, the virus was detected in nasal and lung washes on days 3 and 5 after intranasal inoculation with $10^{6} \mathrm{TCID}_{50}$ of SARS-CoV. Virus antigen was found in the epithelial cells in the lung alveolar and nasal cavities on day 3 as well as slight inflammatory reaction. In contrast, virus antigen was observed in the epithelial cells of intrapulmonary bronchi with inflammatory cells, including macrophages, in F344 rats after intranasal inoculation with $10^{7} \mathrm{TCID}_{50}$ of SARS-CoV (Figure 1D). ACE2 antigen was observed in the epithelial cells of the respiratory tract in mock-infected F344 rats (Figure 1E). In BALB/c mice, ACE2 antigen-positive cells were not detected in noninfected areas (Figure 1F). In these experimental animals, ACE2 antigen-positive cells were observed in the virus-infected area and were repairing swelled type II alveolar epithelium (Table 1). In BALB/c mice and F344 rats, the virus was eliminated by day 7 p.i. None of the three experimental animals developed any clinical symptoms similar to SARS-like disease.

\section{DISCUSSION}

It was suggested that the infection and replication of SARS-CoV occurred in the respiratory tract of these experimental animals. However, there were differences in pathological findings, such as distribution of SARS-CoV and ACE2 antigen in the lungs between monkey, mouse, and rat (Table 1). The localization of infection with SARS$\mathrm{CoV}$ was associated with the distribution of ACE2 antigen. These results indicated that ACE2 was used as a receptor for SARS-CoV infection in these animals. Furthermore, pathological dissimilarities in infectious and inflammatory reactions were observed between BALB/c mice and F334 rats. It was reported previously that monkeys were not suitable for studies of SARS. ${ }^{3,4}$ Although none of the three types of experimental animal examined here developed any SARS-like symptoms, SARS-CoV could infect and replicate in these animals after intranasal or intratracheal inoculation. Therefore, these 
animal models are thought to be useful not only for studies on SARS-CoV infection and pathogenesis, but also for evaluation of novel vaccine and antiviral drugs against this virus.
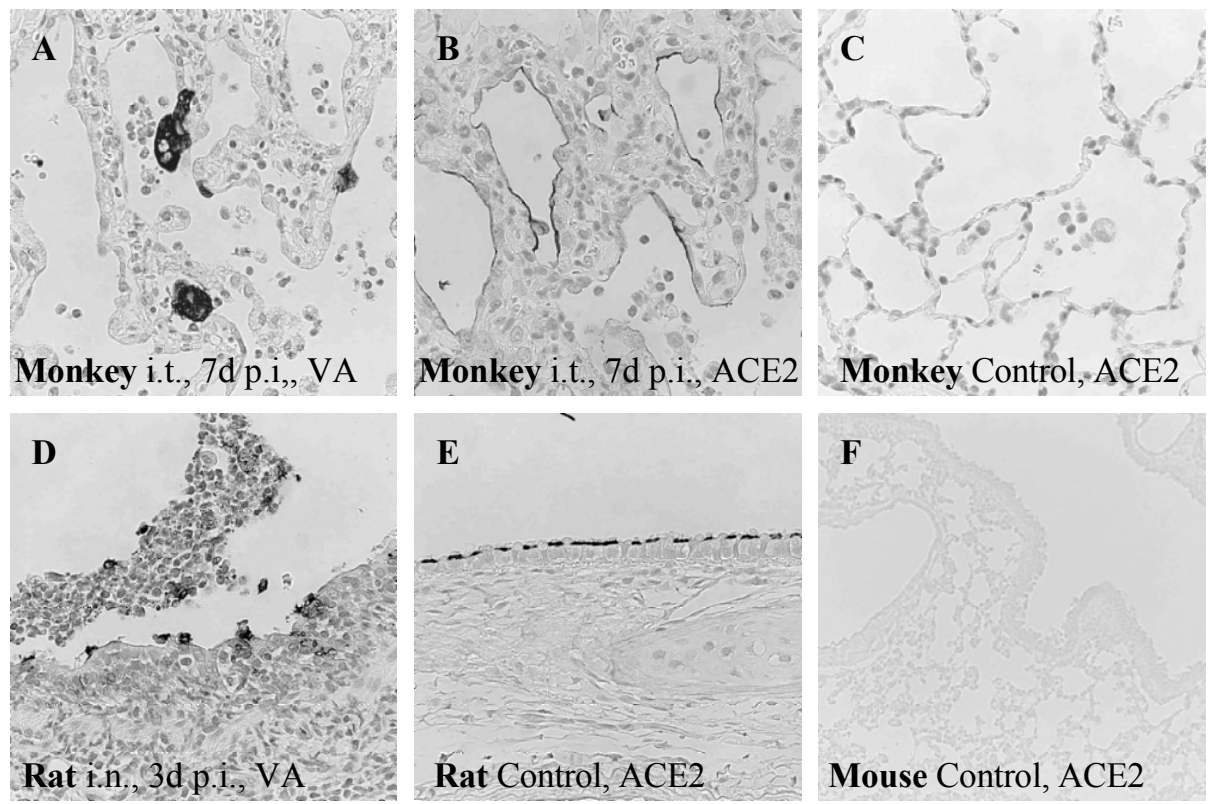

Figure 1. Distribution of SARS-CoV and ACE2 antigens in lung tissue determined using the immunoperoxidase method. VA, virus antigen. Original magnification: A-E, x400; F, x100.

Table 1. Distribution of SARS-CoV antigen and expression of ACE2 in experimental animals.

\begin{tabular}{|c|c|c|c|c|c|c|c|}
\hline & & \multicolumn{3}{|c|}{ Virus antigen } & \multicolumn{3}{|c|}{ Expression of ACE2 } \\
\hline & & Monkey & Mouse & Rat & Monkey & Mouse & Rat \\
\hline \multirow{2}{*}{$\begin{array}{l}\text { Nasal } \\
\text { cavity }\end{array}$} & Respiratory area & - & - & + & - & - & + \\
\hline & Olfactory area & + & + & - & - & - & - \\
\hline \multirow[t]{2}{*}{ Trachea } & Trachea & - & - & + & - & - & + \\
\hline & $\begin{array}{l}\text { Intrapulmonary } \\
\text { bronchi }\end{array}$ & - & - & + & - & - & + \\
\hline \multirow[t]{2}{*}{ Lung } & Bronchioles & - & - & + & - & - & + \\
\hline & Alveoli & + & + & + & $(+)^{*}$ & $(+)^{*}$ & $(+)^{*}$ \\
\hline
\end{tabular}

* ACE2 antigen-positive cells were observed in the virus-infected area and were repairing swelled type II alveolar epithelium. 


\section{ACKNOWLEDGMENTS}

We wish to thank Dr. Joseph S. M. Peiris, Department of Microbiology, the University of Hong Kong, for providing the HKU39849 strain of SARS-CoV. This work was supported by a Grant-in-Aid for Research on Emerging and Re-emerging Infectious Diseases from the Ministry of Health, Labour and Welfare, Japan, and a Grant-in-Aid for Scientific Research from the Ministry of Education, Culture, Sports, Science, and Technology, Japan.

\section{REFERENCES}

1. Peiris, J. S. M., Lai, S. T., Poon, L. L. M., Guan, Y., Yam, L. Y. C., Lim, W., Nicholls, J., Yee, W. K. S., Yan, W. W., Cheung, M. T., Cheng, V. C. C., Chan, K. H., Tsang, D. N. C., Yung, R. W. H., Ng, T. K., Yuen, K. Y., and members of the SARS study group, 2003, Coronavirus as a possible cause of severe acute respiratory syndrome, Lancet 361:1319-1325.

2. Li, W., Moore, M. J., Vasilieva, N., Sui, J., Wong, S. K., Berne, M. A., Somasundaran, M., Sullivan, J. L., Luzuriaga, K., Greenough, T. C., Choe, H., and Farzan, M., 2003, Angiotensin-converting enzyme 2 is a functional receptor for the SARS coronavirus, Nature 426:450-454.

3. McAuliffe, J., Vogel, L., Roberts, A., Fahle, G., Fischer, S., Shieh, W. J., Butler, E., Zaki, S., St Claire, M., Murphy, B., and Subbarao, K., 2004, Replication of SARS coronavirus administered into the respiratory tract of African Green, rhesus and cynomolgus monkeys, Virology 330:8-15.

4. Rowe, T., Gao, G., Hogan, R. J., Crystal, R. G., Voss, T. G., Grant, R. L., Bell, P., Kobinger, G. P., Wivel, N. A., and Wilson, J. M., 2004, Macaque model for severe acute respiratory syndrome, J. Virol. 78:1140111404. 